\title{
Non-destructive Characterization of Micro-sized Defects in the Solar Cell Structure
}

\author{
Bogatyreva Nadezda ${ }^{1, a}$, Macku Robert ${ }^{1, b}$ \\ ${ }^{1}$ Department of Physic, Brno University of Technology, Faculty of Electrical Engineering and \\ Communication, Technicka 8, Brno 61600, Czech Republic \\ ${ }^{a}$ xbogat00@stud.feec.vutbr.cz, ${ }^{\mathrm{b}}$ macku@feec.vutbr.cz
}

Keywords: Solar cell, Microplasma noise, Non-destructive testing, Local defect

\begin{abstract}
This work is focused on noise measurements application for solar cells quality assessment. Particular micro-sized defects are responsible for impulse noise, usually referred to as Microplasma noise. This phenomenon will be investigated in time and frequency domain in this paper. In addition, the Microplasma defect region size is determined using capacitance measurement. Note, that presented non-destructive measurement methodology is suitable for testing of devices with potential barrier not only for solar cells.

\section{Introduction}

Although silicon solar technology is most widespread and production process is relatively cheap, the low efficiency and production cost are still central problems of photovoltaics systems. In order to improve efficiency the new diagnostic methods and defects description are very good approach. Some of defects can be revealed using electric measurement, luminescence techniques, laser/electron beam induced current techniques and electro-ultrasonic spectroscopy, [1]. Structure defects that emit light in visible range while are reversely biased can be precisely localized in microscale using scanning probe microscopy, [2]. In the most cases defects and imperfections are micrometer sized regions. These small regions that can be localized and classified affect parameters of the whole samples, [3]. Imperfections which are optically active, while the sample is in reverse biased condition, can be classified using thermal dependence of light emission, [4].

We introduced the solar cells noise diagnostic methods. Complicated noise signals can be measured as a result of many noise sources (many defects) and large $p n$ junction area. The main problem is separation of defects contribution regardless noise nature. It is suitable for e. g. electrolytic capacitors, resistors or indeed semiconductor devices quality assessment, see [5]. The main advantage is non-destructive character of testing and possibility to make test during ordinary operation of the device.
\end{abstract}

\section{Observed noise signals - Microplasma noise}

As mentioned earlier we can observe number of different noise sources or their superposition respectively. In many cases their nature and reason of origin is unknown. Therefore we use categorizing name notation such as $A$-type or $B$-type etc. The $A$-type noise usually appears at sufficiently high reverse voltage, yet lower than the breakdown voltage of the complete defect-free junction regions, and it is caused by avalanche ionization breakdown. This noise is known as the Microplasma noise and it is the focal point of our study at present. Figure 1a depicts the Microplasma current noise in the time domain. The Microplasma noise appears as a result of local defects with positive temperature dependence of breakdown (critical) voltage, $U_{\mathrm{BR}}$, as depicted in 
Fig. 1b. Thanks to this fact the obtained results may be directly used for non-destructive diagnostics and quality assessment of samples.

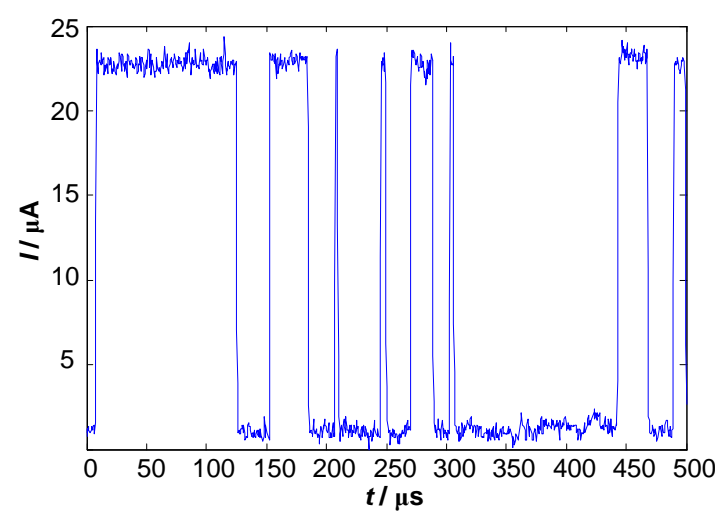

a)



b)

Fig.1. a) Microplasma noise current in the time domain, sample $\mathrm{K} 2, U_{\mathrm{R}}=10.5 \mathrm{~V}, T=25^{\circ} \mathrm{C}$.

b) Temperature dependence of breakdown voltage, sample K4.

Noise in the frequency domain. Particular efforts were devoted to the study of noise in the frequency domain. The Microplasma noise has a generation-recombination noise (G-R noise) character in the frequency domain. G-R noise is caused by free charge carrier fluctuation as a result of carriers quantum transition between the conductive and valence band. It is clear that physical nature of microplasma noise is quite different nevertheless both phenomena produce impulse current fluctuation. The physical interpretation in the sense of avalanche breakdown is again supported by temperature investigations, see Fig. 1b. The experimental results of power spectral density (PSD) measurements are depicted in Fig. 2a.

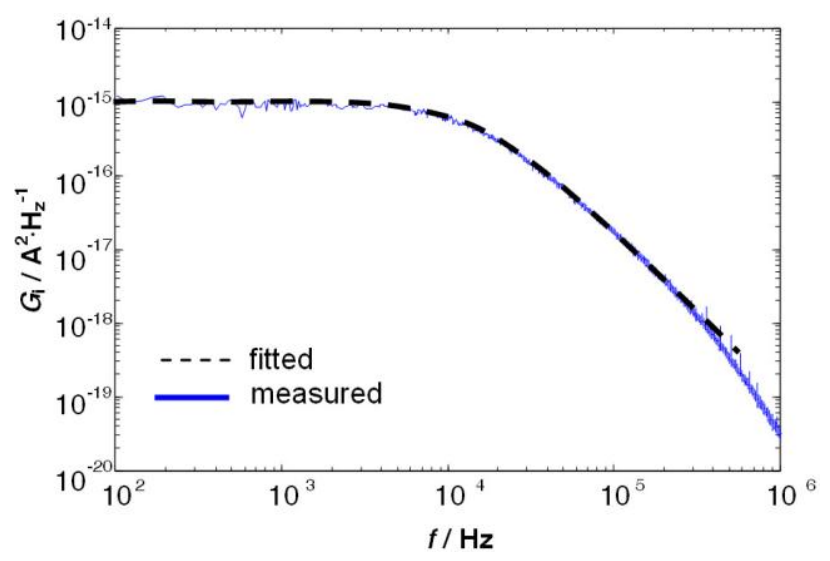

a)

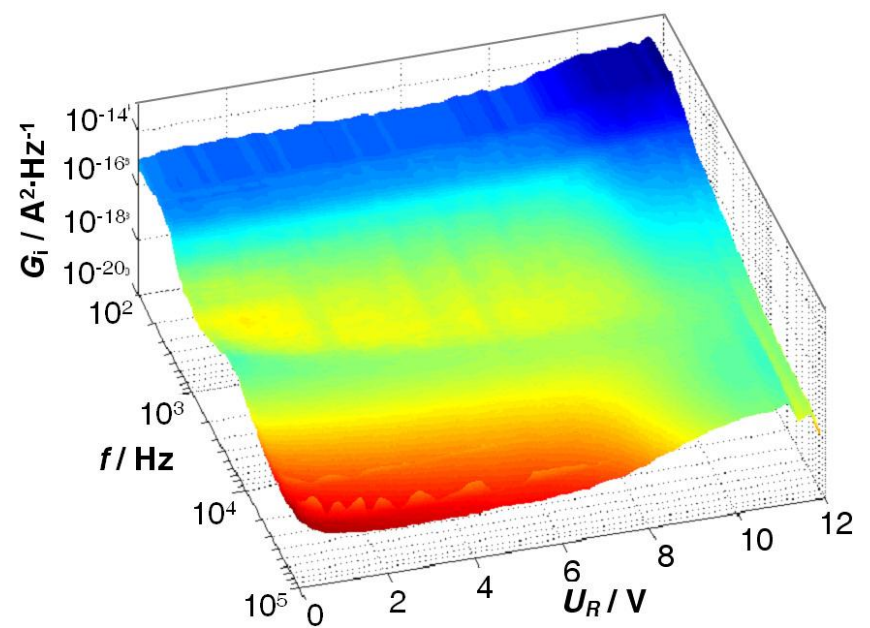

b)

Fig.2. a) Power spectrum density of microplasma noise, sample $\mathrm{K} 2, U_{\mathrm{R}}=10.5 \mathrm{~V}, T=25^{\circ} \mathrm{C}$.

b) Power spectrum density for various reverse voltages, sample 30_3, $T=30^{\circ} \mathrm{C}$.

The presented kind of noise can be mathematically described as the Marcovian Poisson process and it is possible to derive analytical formula based on impulse creation and destruction probability. Therefore, noise spectrum can be analyzed using approximation procedure as shown in Fig. 2a. It should be mentioned that by curve fitting (Fig. 2a) in according to analytical formula (more 
information in Ref. 5) mean value of impulse time duration $t_{1}$ and separation $t_{2}$ may be determined (in case of sample $\mathrm{K} 2$ and $U_{\mathrm{R}}=10.5 \mathrm{~V}, t_{1}=7.7 \cdot 10^{-4} \mathrm{~s}$ and $t_{2}=9.0 \cdot 10^{-3} \mathrm{~s}$ ). Finally it is possible to calculate electrons and holes ionization rate and impact ionization coefficient as a function of electric field.

The power spectrum density has been measured for a wide range of reverse voltages $U_{\mathrm{R}}$ up to $12 \mathrm{~V}$ with fine voltage step $50 \mathrm{mV}$ and spectrum development has been monitored. The experimental results are depicted in Fig. 2b. Noise composition is usually very complicated and we are able to distinguish four noise types now. So, Microplasma noise, Flicker noise, Thermal noise and Shot noise. More information can be found in the dedicated literature e.g. [6]. All of this signal except the Microplasma noise pertinent to bulk processes and inhomogeneties and are left out of consideration in this paper.

Narrow band noise measurement. For purposes non-destructive testing in production industry or/and if we want to known only number of microplasma regions, it is not necessary complicated PSD measurement make. The results indicate that G-R spectrum have not neglectable power in the frequency range where others noises are not significant. This effect consecutively leads to the other measurement philosophy. We can measure only narrowband noise signal on the center frequency e.g. $3 \mathrm{kHz}$ (see Fig. 2a). We use the selective nanovoltmeter with high selectivity and effective noise bandwidth of about $30 \mathrm{~Hz}$. The mean effective value (integration time is $t \approx 3 \mathrm{~s}$ ) of narrowband signal is measured via the DC voltmeter. Figure 3a depicts this experimental obtained narrowband signal. It is interesting to see that microplasma regions are very close bounded and smaller peaks indicate another type of noise presence. It is distinguished by the small junction area (only fragment of a whole solar cell) and due to the fact it is possible to study just only two local defects. For narrow band measurement here was chosen center frequency $3.4 \mathrm{kHz}$.

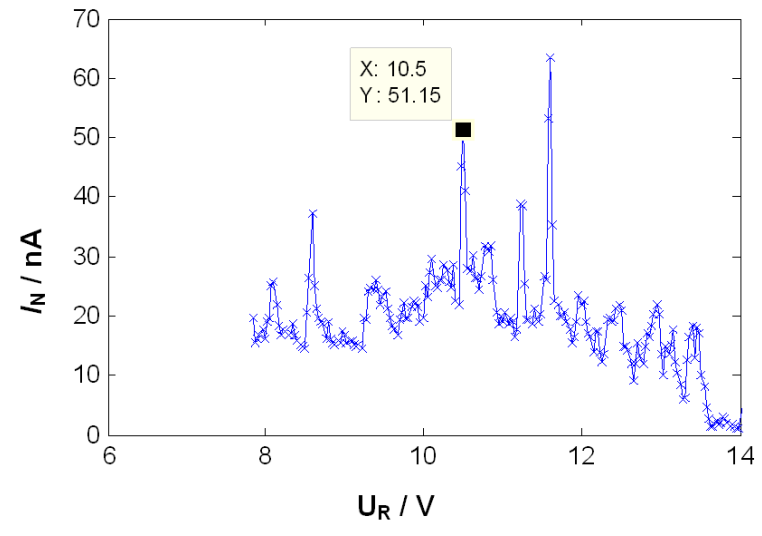

a)

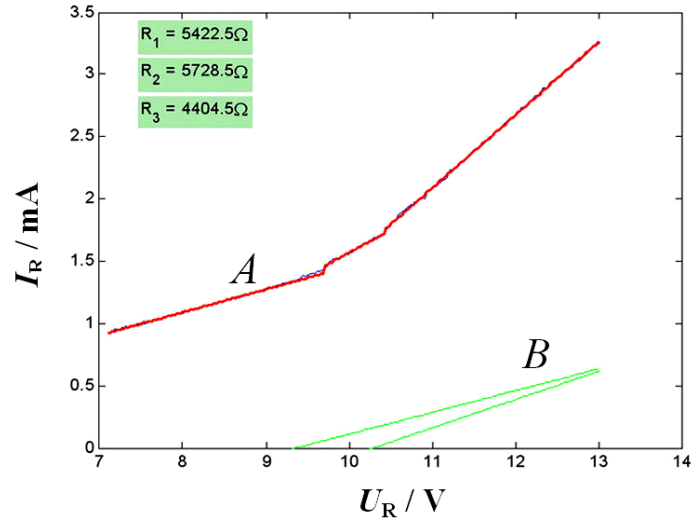

b)

Fig.3. a) Narrowband noise signal for various reverse voltages, sample K2, $T=25^{\circ} \mathrm{C}$. b) I-V curves of reverse-biased solar cell and two local breakdowns, sample K2.

Figure $3 \mathrm{~b}$ illustrates connection of local breakdowns with I-V curves of the solar cell (curve $A$ ). Here two local breakdowns are evident and degree of potential destruction may be specified. The microplasma breakdown is supported by the Microplasma noise presence in the time domain. $R_{2}$ and $R_{3}$ are resistance of conductive channels and the lines $B$ are I-V curves of these channels determined from solar cell I-V curve. The resistor $R_{1}$ represents excess reverse current of the solar cell sample. 


\section{Capacitance characterization of local defects}

We carried out experimental measurement of many reverse-biased samples for different reverse voltage $U_{R}$. Overall majority of solar cells contain defect regions inductive of breakdowns. In general, breakdowns are often classificated by means of $\mathrm{d} U_{R} / \mathrm{d} t$ ratio in the literature. Here $U_{R}$ is the reverse voltage and $t$ is time. If this ratio is relatively small, the breakdown is referred to as quasi-static or static. On the other hand, if the ratio is extremely high, the breakdown is referred as a dynamic. Critical value and more information are possible to find out in the literature, [7].

Qualitative description of reverse biased $p n$ junction I-V curve and breakdown descriptions in sample is in Fig. 4. The first region which can be seen is caused by saturation current incidence. The avalanche multiplication of carries is indicated close to the highlighted rectangle and the blue highlighted rectangle itself represents bi-stable microplasma regions. For higher reverse voltage a bi-stable mechanism disappears and the permanent conductive channel is created.

New local conductive channels may be created for higher reverse voltage and it results in the reliability and the life-time reduction of solar cells. Homogeneous breakdown voltage for defect-free sample is about $U_{B R} \approx 30 \mathrm{~V}$ and $R_{d} \approx 5 \Omega$ (verified on the sample BR1).

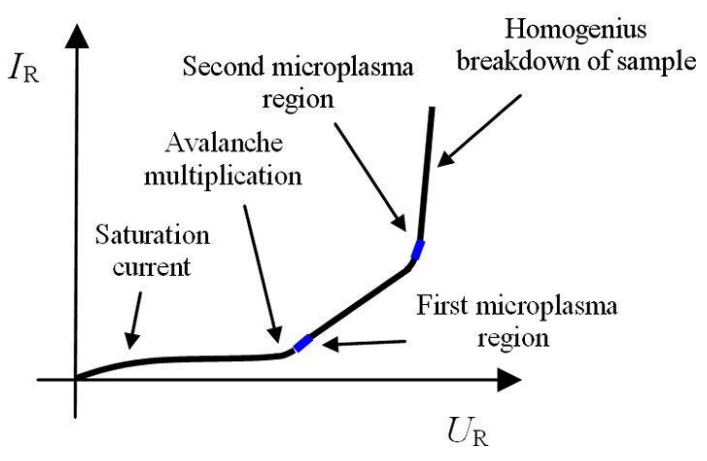

Fig.4. Qualitative I-V curve of reverse biased $p n$ junction of solar cell where microplasma breakdowns are evident.

The Microplasma breakdowns are known for a long time. Back in 1961 Shockley observed local breakdown presence in case of silicon pn junction and in 1964 Haitz put into practice his electric model of breakdown behaviour, [10]. This type of breakdown pointed out imperfections in semiconductor structure. Nevertheless, related experiments must have strictly non-destructive character with high selectivity to particular phenomenon. That is why topical research lack of physical interpretation and defect geometry as well as reason of defects origin is still a question.

Capacitance based local defects properties investigation. The sample $\mathrm{K} 2$ has been selected for study of local defects properties. It is distinguished by the small junction area (only fragment of a whole solar cell) and due to the fact it is possible to study only two local defects. Mechanical properties have been specified by means of calibrated high resolution camera and software based post processing (see Fig. 5a).

It stands to reason that the sample macroscopic area is about $S=92.2 \mathrm{~mm}^{2}$ and the $p n$ junction area is about $S_{\text {jun }}=134.6 \mathrm{~mm}^{2}$ (the surface texturization is under consideration). The $\mathrm{C}-\mathrm{V}$ curves of the sample $\mathrm{K} 2$ has been measured under the same conditions as a noise characteristic presented before. It turns out that the $p n$ junction concentration profile acts as an abrupt step and it is very good controlled by the production technology at present. In the voltage interval where the bi-stable noise process is active and the avalanche multiplication occurs is impossible to make accurate capacitance 
measurement. That is why the $\mathrm{C}-\mathrm{V}$ curve is not in continues form, see Fig. 5b. The first linear section has been fitted (approximately up to $U_{\mathrm{R}}=9.5 \mathrm{~V}$ ) and the acceptor concentration has been determined. The obtained value is $N_{2}=5.85 \cdot 10^{21} \mathrm{~m}^{-3}$ (rezistivity $\rho \approx 3 \Omega \cdot \mathrm{cm}$ ).

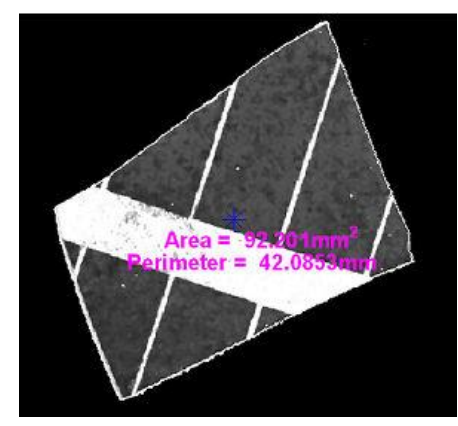

a)

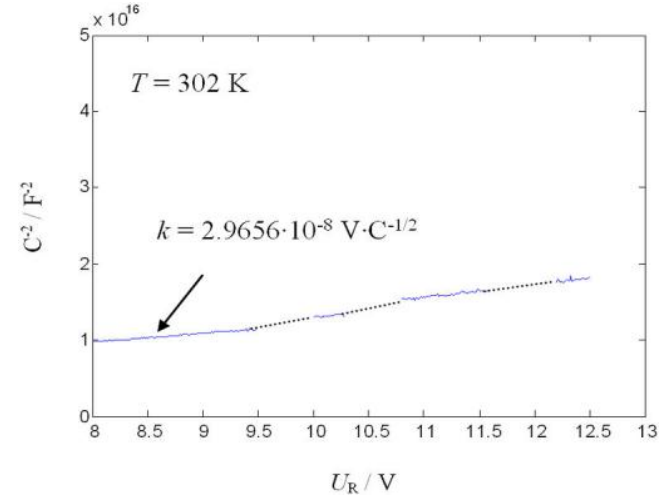

b)

Fig.5. a) Sample of solar cell (K2) and software analysis of geometric properties. b) The C-V characteristic of the solar cell (K2) which includes local defects.

In a broad sense, we can suppose that the $p n$ junction is approximately plane and its capacitance is given by equation for the plate condenser

$C=\frac{\varepsilon_{r} \varepsilon_{0} S}{d}$

We can determine behaviour of maximum electric field intensity in the junction $\left(E_{\mathrm{max}}\right)$ by means of equation electric intensity (2)

$E_{\max }=\frac{e N_{1} d_{1}}{\varepsilon_{r} \varepsilon_{0}}=\frac{e N_{2} d_{2}}{\varepsilon_{r} \varepsilon_{0}}$,

as a function of reverse voltage value (see Fig. 11). It should be noted that the sharp peaks (see Fig. 5) again result from the bi-stable microplasma breakdowns and they do not represent valid electric intensity because of random current fluctuation.

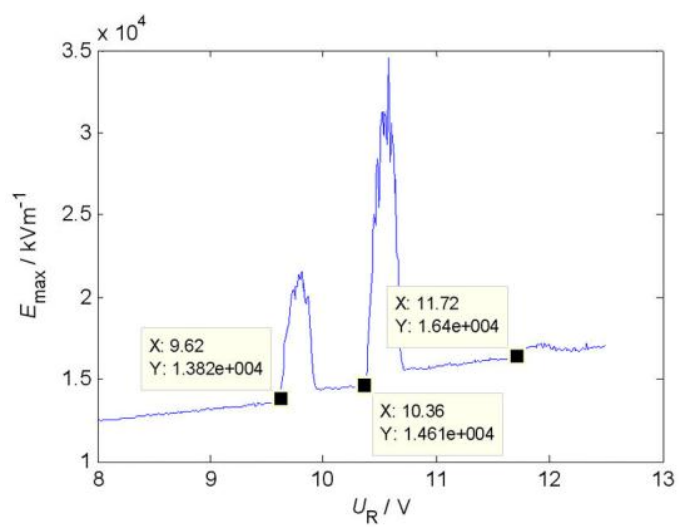

Fig.5. Maximum electric intensity in $p n$ junction which includes local defects (local breakdowns), solar cell (K2). 
Nevertheless, it may be useful to boundary bi-stable noise process. Otherwise we can exactly determine when breakdown of the $p n$ junction occurred.

Technically, we can also suppose that the local breakdowns of a silicon sample are in small scale similar as homogeneous breakdowns. This assumption is in agreement with measurement of thermal characteristics (breakdown voltage as a function of temperature, see Fig. 1b). Enough experimental equation for the critic (breakdown) voltage in silicon as a function impurities concentration is reported in the literature such as [9], [10]. Familiar equation for description of breakdown voltage vs. impurities concentration in the silicon finds out for example Miller and Shields [11], [12]. Another empirical formula, which is derived for the non-symmetrical abrupt junction follows (more in [9])

$U_{B}=60\left(\frac{W_{g}}{1.1}\right)^{3 / 2}\left(\frac{N_{A}}{10^{16}}\right)^{-3 / 4} \quad\left(\mathrm{~V} ; \mathrm{eV} ; \mathrm{cm}^{-3}\right)$

Here $U_{\mathrm{B}}$ is breakdown voltage, $W_{\mathrm{g}}$ is forbidden band width and $N_{\mathrm{A}}$ is acceptor concentration. Decrease of breakdown voltage in defect region compared to the total homogeneous breakdown may be caused for example by increase of acceptor concentration in the wafer. This effect may be caused by aggregation of impurity atoms in the wafer during cool-down process.

Let us consider slightly high acceptor concentration in range $N_{\mathrm{A}}=\left(10^{17} \div 10^{19}\right) \mathrm{cm}^{-3}$ and temperature $T=300 \mathrm{~K}$, then the forbidden band energy of silicon is approximately $W_{\mathrm{g}}=1.13 \mathrm{eV}$. The next assumption is that the defect region has an effect on the $p n$ junction barrier and variation of width is caused by variation of wafer acceptor concentration (schematically depicted in Fig. 6.).

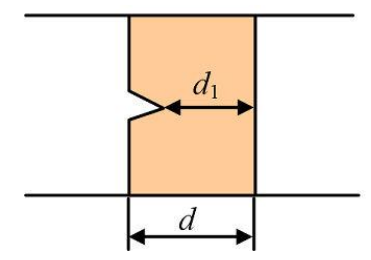

Fig.6. Qualitative model of $p n$ junction with defect, $d$ is depletion region width; $d_{l}$ is depletion region width in defect region

Let us consider the first defect region as we can see in Fig. 3b or Fig. 5. The diffusion voltage is possible to specify from $\mathrm{C}-\mathrm{V}$ measurement as $U_{\mathrm{DIF}}=0.567 \mathrm{~V}$. The barrier voltage (the breakdown voltage respectively) is $U_{\mathrm{B}}=U_{\mathrm{R}}+U_{\mathrm{DIF}}=9.62+0.567=10.187 \mathrm{~V}$. Here $U_{\mathrm{VN}}$ is voltage of external source. We can calculate the impurity concentration in the first defect region (4) by means of equation (3)

$N_{A}=\left(\frac{U_{B}}{60\left(W_{g} / 1.1\right)^{3 / 2}}\right)^{-4 / 3} \cdot 10^{16}=1.124 \cdot 10^{23} \mathrm{~m}^{-3}$. 
For abrupt junction it holds:

$$
U \approx \frac{e N_{A} d_{1}^{2}}{2 \varepsilon_{0} \varepsilon_{r}} \rightarrow d_{2}=\left(\frac{2 \varepsilon_{0} \varepsilon_{r} U_{B}}{e N_{A}}\right)^{1 / 2},
$$

and the defects region width is $d_{2}=0.34 \mu \mathrm{m}$. Now, we know concentration and moreover the defect region width. Detailed analysis is possible to make for all defect regions in the sample (see Fig. 3b or Fig. 5). Obtained values are summarized in the Table 1.

Table 1. Physical properties of microplasma local defects

\begin{tabular}{|l|c|c|c|}
\hline Breakdown voltage $U_{\mathrm{B}}[\mathrm{V}]$ & 9.62 & 10.36 & 11.72 \\
\hline $\begin{array}{l}\text { Defect-free depletion region } \\
\text { width }[\mu \mathrm{m}]\end{array}$ & 1.50 & 1.55 & 1.65 \\
\hline Defect region size $[\mu \mathrm{m}]$ & 0.34 & 0.37 & 0.43 \\
\hline $\begin{array}{l}\text { Defect region impurity } \\
\text { concentration } 10^{23}\left[\mathrm{~m}^{-3}\right]\end{array}$ & 1.12 & 1.02 & 0.874 \\
\hline
\end{tabular}

\section{Summary}

Our research deals with the non-destructive testing of the silicon solar cells. We carried out number of experiments related to the meso-scale and mirco-scale defect regions. It turns out that is necessary to study bulk and edges imperfections separately because of different specific parameters. This paper discusses small cross-section microplasma defects and related electrical characteristics.

We observe typically local breakdown accompanied by the rectangular Microplasma noise in the time domain. Number of defects affecting the solar cell sample is enormous. Thank to this fact, special afford must be directed to the sample preparation and edge isolation. In order to understanding defects behaviour we study electrical noise in the time domain where random impulse process was indentified. Current fluctuation amplitude is in micro-amps order for voltage bias conditions. On the other hand if current bias is used then breakdown events are accompanied by negative differential resistance regions. Temperature dependence of the breakdown voltage put forward physical interpretation in the sense of impact ionization and local avalanche breakdowns. The Microplasma noise as a random process is characterized analogous to generation-recombination processes and the power spectral density decrease proportionally to frequency squared. We study both narrowband bias voltage interval and power spectral density development with voltage bias. It turns out, that bulk inhomogeneties give a rise of $1 / f$ (flicker) noise process.

In order to find microplasma defect properties we also study $\mathrm{C}-\mathrm{V}$ curves. One of the measurement results is the fact, that the $p n$ junctions of solar cells have a strongly non-symmetrical concentration profile. That is why we can introduce one-sided concentration approximation. This approach makes possible to determine for example acceptor impurities concentration in the substrate and defect region. We study number of samples and we can conclude that acceptor concentration is always just about $N_{2}=1.2 \cdot 10^{21} \mathrm{~m}^{-3}$. It means that the production technology is very well controlled. If local defects are present in a small number, they can affect the measurement capacitance characteristics by the explicit way. In general, we can compute the maximum electric field intensity in samples by means of $\mathrm{C}-\mathrm{V}$ characteristic. We introduce the local defects which act as a pn junction but in contrast to a defect-free region they have a different impurities concentration. Therefore this $p n$ junction may be characterized by different breakdown voltage. Local defects have always higher 
impurity concentration as shown in Table 1. It may be caused by precipitates or admixtures. This situation consequently leads to the inhomogeneous $p n$ junction creation and reduction of quality.

\section{Acknowledgements}

This paper is based on the research supported by the Grant Agency of the Czech Republic, the grant No. P102/10/2013, on the research supported by the Ministry of Industry and Trade of the Czech Republic, the project MPO TIP FR-TI1/305 and on the SIX research centre (project registration number: CZ.1.05/2.1.00/03.0072).

\section{References}

[1] P. Tofel, J. Sikula, K. Hajek, Z. Trojanova, L. Bumbalek: Key Eng. Mat., vol. 465 (2011), p. 350-353.

[2] P. Skarvada, P. Tomanek, L. Grmela and S. Smith: Sol. Energ. Mat. Sol. C., Vol. 94 (2010), p. 2358-2361.

[3] A. Namajunas, A. Tamasevicius, G. Nykolaitis, S. Bumeliene, J. Pozela: Acta Physica Polonica A, vol. 107 (2011), p. 369-372.

[4] L. Grmela, P. Skarvada, P. Tomanek, R. Macku, S. Smith: Sol. Energ. Mat. Sol.C., Vol. 96 (2012), p. 108-111.

[5] P. Koktavy, J. Sikula: Fluct. Noise Lett.,Vol. 2 (2002), p.L65-L70.

[6] A. Van Der Ziel: Noise in solid state devices and circuits, John Wiley \& Sons (1986).

[7] M. Levinshtein, J. Kostamovaara, and S. Vainshtein: Breakdown phenomena in semiconductors and semiconductor devices, World Scientific Publishing, Singapore (2005).

[8] R.H. Haitz: J. Appl. Phys. Vol. 35, No. 5, (1964), p. 1370-1376.

[9] S.M. Sze: Physics of Semiconductor Devices, John Wiley \& Sons, NY, (November 2006).

[10] P.Y. Yu, M. Cardona: Fundamentals of Semiconductors: Springer, (2010).

[11] S.L. Miller: Phys. Rev., vol. 105, p. 1246-1249 (1957).

[12] J. Shields: Journ. Electron. Control, vol. 6, p. 132-148, (1959). 